\title{
Kinesin superfamily protein member 4 (KIF 4 ) is localized to midzone and midbody in dividing cells
}

\author{
Young Mi Lee ${ }^{1}$ and Wankee $\mathrm{Kim}^{1,2}$ \\ ${ }^{1}$ Institute for Medical Sciences \\ School of Medicine, Ajou University \\ Suwon 442-749, South Korea \\ ${ }^{2}$ Corresponding author: Tel, 82-31-219-4506; \\ Fax, 82-31-219-4503; E-mail, wkim@ajou.ac.kr \\ Accepted 16 February 2004
}

Abbreviations: DAPI, 4',6-diamidino-2-phenylindole; DMEM, Dulbecco's minimum essential media; FITC, Fluorescein isothiocyanate; KIF4, kinesin superfamily protein member 4

\begin{abstract}
In association with microtubules, a variety of kinesins play important roles in cellular functions such as intracellular transport of organelles or vesicles, signal transduction, and cell division. In a previous study we revealed that human kinesin superfamily protein member 4 (KIF4) is a chromokinesin that binds to chromosomes. Since localization of several kinds of kinesin at midzone called central spindle, or midbody that connects two daughter cells, or both, suggests their implication in cell division, we investigated KIF4 localization of during mitosis and cytokinesis in Hela cells. In addition to association with segregating chromosomes through entire mitosis, it also localized to the midzone and to midbody at ana/telophase through cytokinesis. Especially in cells at cytokinesis, KIF4 appeared as a doublet facing each other at the apical ends of two daughter cells. Threedimensional analysis of architectural relationship between microtubule bundles and KIF 4 indicated that KIF4 forms a ring structure wrapping around the microtubule bundles. These results suggest that KIF4 is involved in cytokinesis, although direct evidence was not provided in this study.
\end{abstract}

Keywords: cell division; chromosome; kinesin; mitosis; segregation; structure

\section{Introduction}

Microtubule-dependent movement carried out by two superfamily motor proteins called cytoplasmic dynein and kinesin is fundamental to many biological processes including cell division and intracellular transport of membranous organelles in higher eukaryotic cells (Hirokawa, 1998; Rieder and Salmon, 1998; Heald and Walczak, 1999). Kinesin or kinesin-like proteins have highly conserved motor domains responsible for microtubule binding and ATP hydrolysis for movement along microtubules. In contrast, no significant sequence similarity is detected in the coiled-coil stalk and cargo-binding tail domains. The diversity of kinesin suggests that each kinesin may have a specific function.

According to the recent phylogenetic analysis of kinesins (http://www.blocks.fhcrc.org/ kinesin/), kinesin superfamily member 4 (KIF4) includes 4 each vertebrate [human and murine KIF4s, chick chromokinesin, and Xenopus KLP1(Xklp1)] and invertebrate (KLP31D and KLP3A of Drosophila, and T01G1 and Y43F4B of $C$. elegans) kinesins. Chick chromokinesin with DNA binding activity is a nuclear protein and associates with chromosome arms (Wang and Adler, 1995), and Xklp1, which is considered to be a nuclear protein, positions mitotic chomosome and stabilizes bipolar mitotic spindle (Vernos et al., 1995). Murine KIF4 is found in both cytoplasm and nucleus, and colocalizes with membranous organelles in interphase cells and with spindle microtubules in mitotic cells (Sekine et al., 1994). It also has been shown to be involved in the anterograde transport of vesicles containing $L 1$, a cell adhesion molecule implicated in axonal elongation (Peretti et al., 2000). Previously we found that human KIF4 of 1,232 amino acids is a chromokinesin (Lee et al., 2001). Although it is revealed that human KIF4 interacts with BRCA2-associated factor 35 in a complex of $540 \mathrm{kDa}$ (Lee and Kim, 2003), its cellular function (s) remains undefined.

Protein components of chromosomal passenger complex associate with kinetochores during early stages of mitosis and dissociate from them as the chromosomes segregate and then localize to midzone at ana/telophase (Adams et al., 2001), contrasting molecules such as kinesin-like motor proteins that simply localize to the central spindle (Field et al., 1999). We have been interested in localization of human KIF4 during the late stages of mitosis, although its other locations beside of chromosomes during mitosis were not noticed in the mitotic cells (Lee et al., 2001). With KIF4 antibodies having a different epitope specificity, we could clearly envision its localization to midzone called central spindle 
during anaphase and to midbody connecting two daughter cells during cytokinesis.

\section{Materials and Methods}

\section{Cell cultures}

HeLa cells were grown in DMEM supplemented with $10 \%$ FBS.

\section{Antibodies and Western blot analysis}

The C-terminal portion of human KIF4 (amino acids 858-1232) was fused with HisTag. After induction with IPTG, inclusion body fraction that harbors most His-tag-KIF4 fusion proteins was concentrated and used for purification of immunogen. Following the standard immunization procedures, mouse and rabbit polyclonal KIF4 antibodies were affinity-purified as described (Lee et al., 2001). Anti-a-tubulin monoclonal antibody (AB-1) was purchased from Oncogene (San Diego, CA). Western blot analysis was performed as described elsewhere (Jee et al., 2003).

\section{Immunocytochemistry}

Mitotic cells were concentrated on a poly-L-lysinecoated coverglass by treating growing cells with nocodazole $(0.3 \mathrm{mg} / \mathrm{ml})$ for $16 \mathrm{~h}$. Coverslips containing cells released from nocodazole for $0,45,90$, and 180 min were washed, fixed, and then permeabilized with $0.3 \%$ Triton X-100 in PBS. After brief washes, coverslips were incubated with the blocking solution $(2 \%$ BSA in PBS) and subsequently with affinity-purified

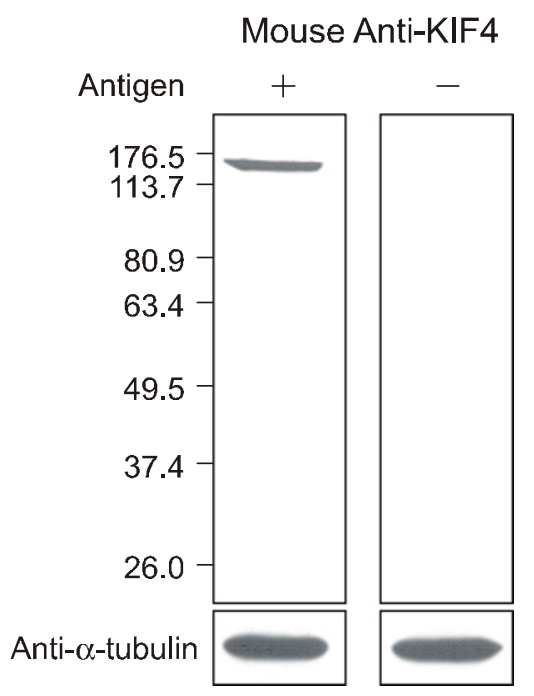

anti-KIF4 antibody and anti-a-tubulin monoclonal antibody as primary antibodies at 1:100 and 1:500 dilution, respectively. FITC-conjugated affinipure goat anti-rabbit $\lg G(H+L)$ and Texas Red-conjugated goat affinipure anti-mouse $\lg G(\mathrm{H}+\mathrm{L})$ (Jackson ImmunoReseach Lab, West Grove, PA) were used as secondary antibodies. Nuclei were counterstained with $0.2 \mathrm{mg} / \mathrm{ml}$ of 4',6-diamidino-2-phenylindole (DAPI). Fluorescence was observed with a confocal laser scanning microscope (LSM510, Zeiss, Thornwood, NJ).

\section{Results and Discussion}

\section{Specificity of KIF4 antibodies}

The KIF4 antibody used in this study was newly prepared from the 374 animo acids from C-terminal of KIF4 molecules fused with His-Tag and purfied from the abundantly expressed inclusion body. This new antibody showed a high titer and different antigenic specificity than those antibody used in an earlier study prepared from the soluble cytosolic proteins from bacterial cell lysates (Lee et al., 2001). Western blot analysis using purified mouse and rabbit KIF4 antibodies showed a single band of $140 \mathrm{kDa}$ in the HeLa cell extract (Figure 1).

\section{Localization of KIF4 in mitotic cells}

In an effort to define whether KIF4 molecules reside only in the chromosomes or localize to other subcellular loci during or after chromosome segregation, the

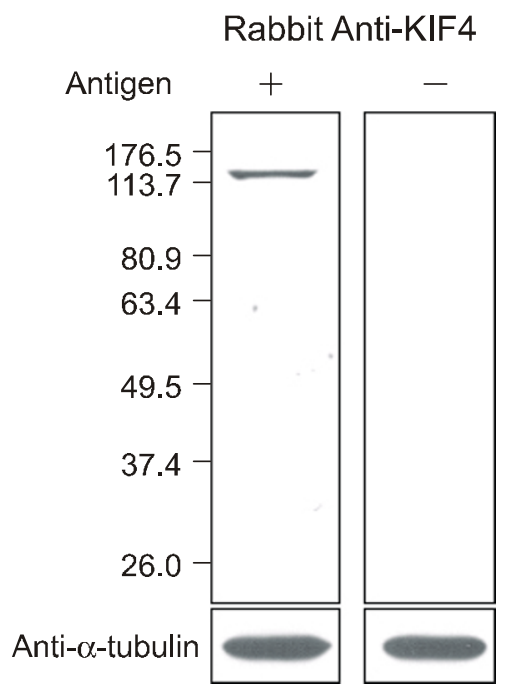

Figure 1. Specificity of antibodies. HeLa cell extracts were separated by SDS-PAGE, transferred to a nitrocellulose filter, and probed with mouse (A) and rabbit (B) anti-KIF4 polyclonal antibodies in the absence (lane 1) or presence (lane 2) of antigen. Anti--tubulin monoclonal antibody was used as a positive control. A single band of $140 \mathrm{kDa}$ was detected. 


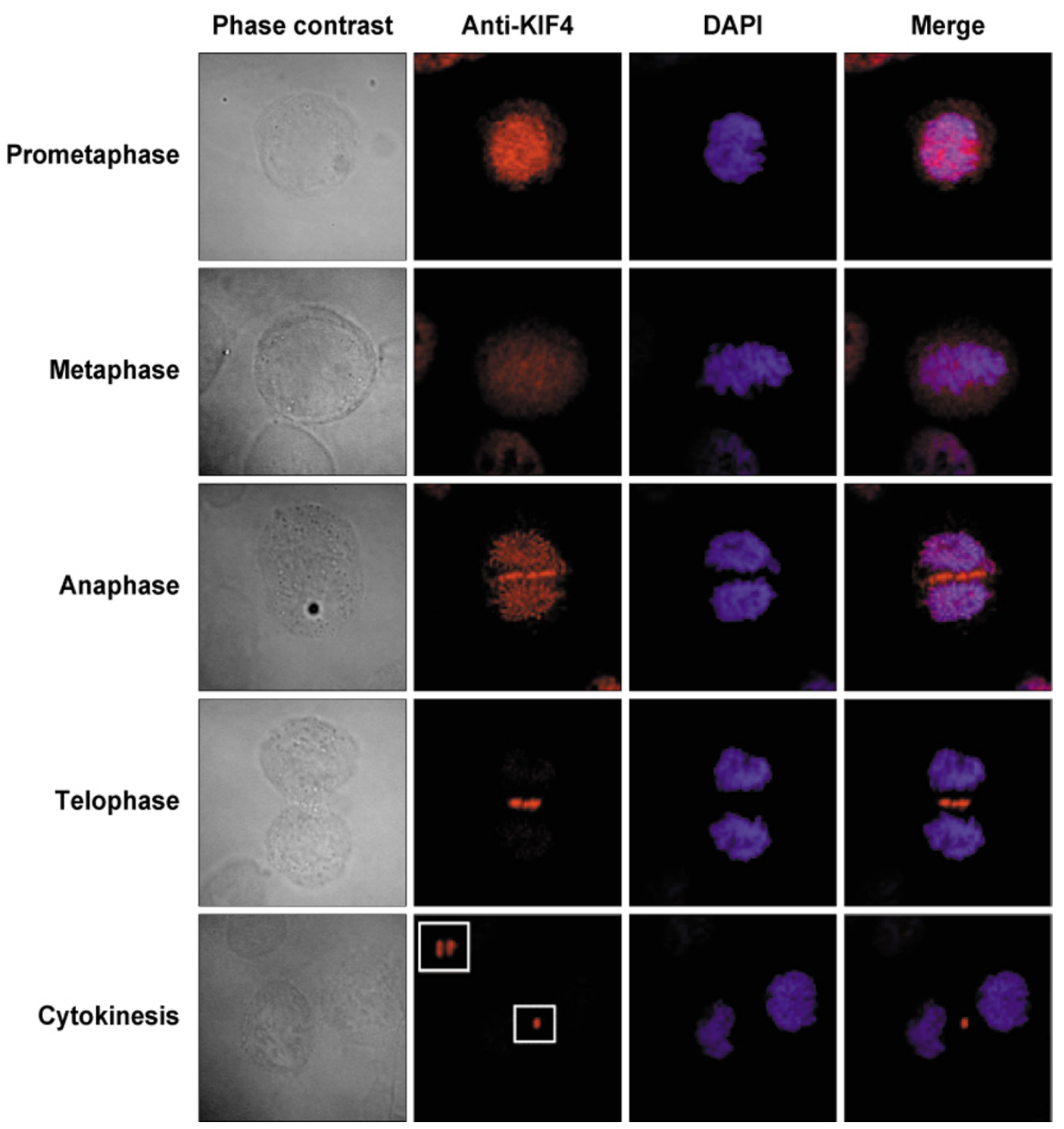

Figure 2. Localization of KIF4 in mitotic cells. The HeLa cells treated with nocodazole (16 h) were released for $0,45,60,90$, and $180 \mathrm{~min}$. to obtain the different stages of mitotic cell. Cells were fixed, permeabilized, and stained with the mouse anti-KIF4 polyclonal antibody as primary antibody (1:100). Texas Red-conjugated goat affinipure anti-mouse $\lg G$ was used as a secondary antibody. Nuclei were counterstained with 0.2 $\mathrm{mg} / \mathrm{ml}$ of DAPI. The insert in cytokinesis is higher magnification of the boxed portion of the image. Some portion of KIF4 associates with chromosome through entire stages of mitosis and other localizes to midzone and midbody.

mitotic cells were synchronized roughly at various stages of prometaphase through near completion of cytokinesis by treating growing cells with nocodazole and release for appropriate time of periods. As shown in Figure 2, when those cells were examined for localization of KIF4 with mouse anti-KIF4 antibody, it initially associated with chromosomes of prometa/metaphase. As cells enter into anaphase, some portion of KIF4 appeared at the central spindle with other still associating with segregating chromosomes. Then, in accordance with shrinkage of the cleavage furrow, a band of KIF4 became compact at telophase and eventually appeared as a dot to midbody bridging two daughter cells at cytokinesis. However, when magnified, this dot turned out to be a doublet facing each other at the apical ends of two daughter cells. Of kinesin-like proteins that are known to be required for cytokinesis, recently identified $\mathrm{M}$ phase phosphoprotein 1 exhibits similar pattern of localization as a doublet at midbody (Abaza et al., 2003).

We did not observe this pattern of KIF4 localization in a previous study (Lee et al., 2001) where KIF4 only associates with segregating chromosomes through mitosis. The difference between the previous and 
present studies was the source of immunogen to produce KIF4 antibody; bacterial cytoplasmic HisTag-KIF fusion protein was used previously whereas fusion protein abundantly present in inclusion body was used here. Although not assured, it is likely that some epitopes of immunogen prepared from bacterial inclusion body may provide additional antigenic epitope sites for recognizing KIF4 localized to midzone and midbody.

\section{Architecture of midbody}

Since cellular functions of kinesin are based on microtubules, we further examined the relationship between KIF4 and microtubules. When two types of cells at early and late cytokensis were stained with rabbit anti-KIF4 and anti-a-tubulin monoclonal antibodies, KIF4 molecules were found positioned at the center of microtubule bundles connecting daughter cells (Figure 3).

It is of additional interest how KIF4 molecules and microtubules were arranged in the midbody. When cells of early cytokinesis were observed from two additional perpendicular angles with higher magnification, a 3-dimensional image showing architectural relationship between KIF4 and microtubules was obtained (Figure 4). Near cross section of midzone microtubule showed a cylindrical structure, whereas KIF4 exhibited crescent-shapes from all directions on the surface of microtubule bundles, indicating a ring structure wrapping around the microtubule bundles.

In addition to assembly of the actomyosin-based contractile ring, a number of proteins that localize to midzone, or midbody, or both, are known to be required for cytokinesis in higher eukaryotic cells. Those identified in early studies include INCENP, survivin, the kinesins CHO-1/MKLP-1, CENP-E, KLP-3A, members of the Polo and aurora kinase families (Field et al and references therein). Recently, several lines of evidence that some kinesins are implicated in cytok-

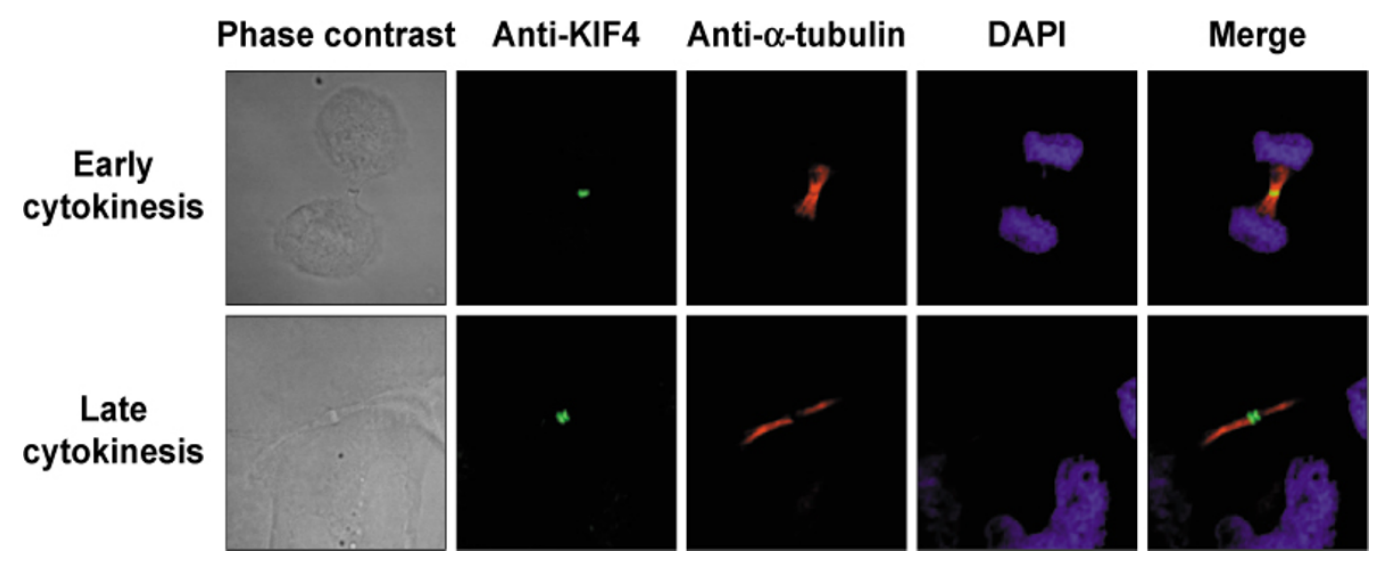

Figure 3. Localization of KIF4 during cytokinesis. Cells at cytokinesis were double-stained with the rabbit anti-KIF4 polyclonal and mouse anti-a-tubulin monoclonal antibodies. FITC-conjugated affinipure goat anti-rabbit IgG and Texas Red-conjugated goat affinipure anti-mouse IgG were used as secondary antibodies. Nuclei were stained with DAPI.
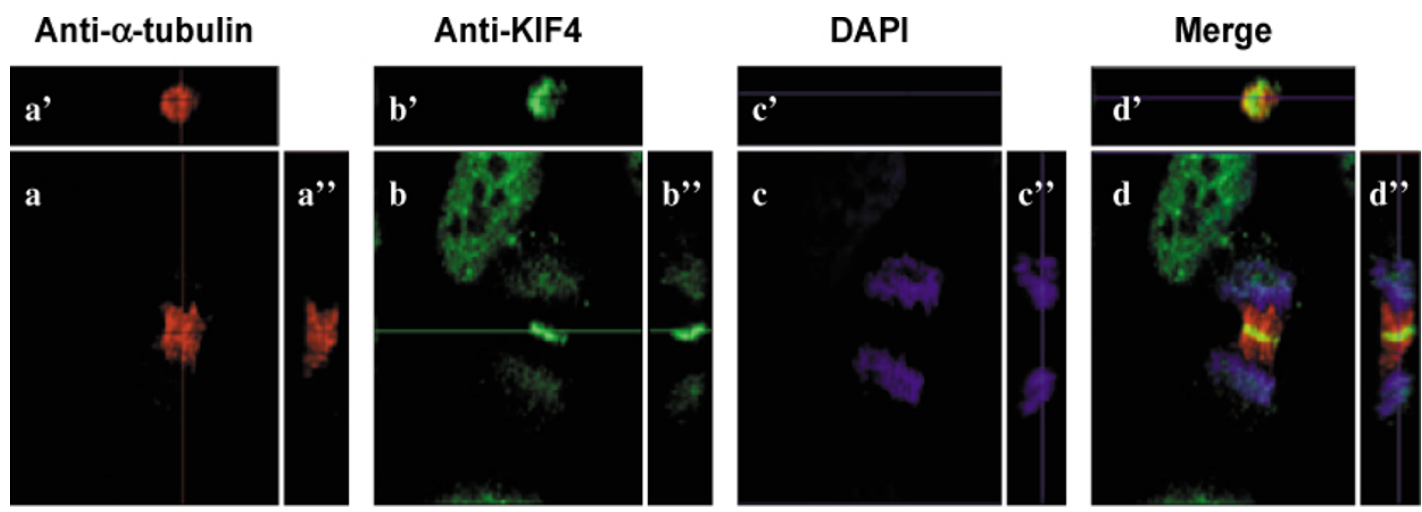

Figure 4. Architectural relationship between KIF4 and microtubules. To determine how KIF4 and microtubule bundles are arranged relatively, 3-dimensional images of midbody were obtained by viewing cells of cytokinesis from front $(a, b, c, d)$, top $\left(a^{\prime}, b^{\prime}, c^{\prime}, d^{\prime}\right)$, and side $\left(a^{\prime \prime}, b^{\prime \prime}, c^{\prime \prime}, d^{\prime \prime}\right)$. 
inesis have been described (Raich et al., 1998; Hill et al., 2000; Matuliene and Kuritama, 2002). However, these are associated with midzone or midbody or both, but not with chromosomes. As shown above, while some portion of KIF4 associated with chromosomes at the initial stages of mitosis, other portion congregated at midzone and further became compact at midbody. These observations differentiate KIF4 from other kinesins that are involved in cytokinesis and chromosomal passenger proteins (Adams et al., 2001) that dissociate from chromosomes and then move to the midzone and midbody. In this regard, KIF4 may perform diverse functions such as DNA segregation and cytokinesis. We suspect that protein modification or interaction(s) with other protein(s) might be responsible for such differential localization of KIF4.

Taken together, KIF4 is suggested to be involved in cytokinesis and further study on the direct evidence will be forthcoming.

\section{Acknowledgement}

This study was supported by a grant of the Korea Health 21 R\&D Project (02-PJ1-PG3-20901-0009), Ministry of Health and Welfare, Republic of Korea.

\section{References}

Abaza A, Soleilhac JM, Westendorf J, Piel M, Crevel I, Roux A, Pirollet F. M phase phosphoprotein 1 is a human plusend-directed kinesin-related protein required for cytokinesis. J Biol Chem 2003;278:27844-52

Adams RR, Carmena M, Earnshaw WC. Chromosomal passengers and the (aurora) ABCs of mitosis. Trends Cell Biol 2001;11:49-54

Field C, Li R, Oegema K. Cytokinesis in eukaryotes: a mechanistic comparison. Curr Opin Cell Biol 1999;11:68-80

Heald R, Walczak CE. Microtubule-based motor function in mitosis. Curr Opin Struct Biol 1999;9:268-74
Hill E, Clarke M, Barr FA. The Rab6-binding kinesin, Rab6$\mathrm{KIFL}$, is required for cytokinesis. EMBO J 2000;19:5711-9

Hirokawa N. Kinesin and dynein superfamily proteins and the mechanism of organelle transport. Science 1998;279:519-26

Jee B, Jin K, Hahn JH, Song HG, Lee H. Metastasissuppressor KAI1/CD82 induces homotypic aggregation of human prostate cancer cells through Src-dependent pathway. Exp Mol Med 2003;35:30-7

Lee YM, Lee S, Lee E, Shin H, Hahn H, Choi W, Kim W. Human kinesin superfamily member 4 is dominantly localized in the nuclear matrix and is associated with chromosomes during mitosis. Biochem J 2001;360:549-56

Lee YM, Kim W. Association of human kinesin superfamily protein member 4 with BRCA2-associated factor 35 . Biochem J 2003;374:497-503

Matuliene J, Kuriyama R. Kinesin-like protein $\mathrm{CHO} 1$ is required for the formation of midbody matrix and the completion of cytokinesis in mammalian cells. Mol Biol Cell 2002; 13:1832-45

Peretti D, Peris L, Rosso S, Quiroga S, Caceres A. Evidence for the involvement of KIF4 in the anterograde transport of L1-containing vesicles. J Cell Biol 2000;149:141-52

Raich WB, Moran AN, Rothman JH, Hardin J. Cytokinesis and midzone microtubule organization in Caenorhabditis elegans require the kinesin-like protein ZEN-4. Mol Biol Cell 1998:9:2037-49

Rieder CL, Salmon ED. The vertebrate cell kinetochore and its roles during mitosis. Trends Cell Biol 1998;8:310-8

Sekine $\mathrm{Y}$, Okada $\mathrm{Y}$, Noda $\mathrm{Y}$, Kondo $\mathrm{S}$, Aizawa $\mathrm{H}$, Takemura R, Hirokawa N. A novel microtubule-based motor protein (KIF -4) for organelle transports, whose expression is regulated deveolpmentally. J Cell Biol 1994;127:187-201

Vernos I, Raats J, Hirano T, Heasman J, Karsenti E, Wylie C. Xklp1, a chomosomal Xenopus kinesin-like protein essential for spindle organization and chomosome positioning. Cell 1995;81:117-27

Wang SZ, Adler R. Chomokinesin: a DNA-binding, kinesinlike nuclear protein. J Cell Biol 1995;128:761-68 